\title{
Innovative Prospects for The Creation of Medical Products Based on The Polysaccharide Matrix
}

\author{
Shelepov VG* and Mager SN \\ Siberian Federal Scientific Center for Agrobiotechnology of the Russian Academy of Sciences, Russia
}

*Corresponding author: Shelepov VG, Siberian Federal Scientific Center for Agrobiotechnology of the Russian Academy of Sciences, Novosibirsk, Russia

\section{ARTICLE INFO}

Received: 幽 February 19, 2020

Published: 幽 February 25, 2020

Citation: Shelepov VG, Mager SN. Innovative Prospects for The Creation of Medical Products Based on The Polysaccharide Matrix. Biomed J Sci \& Tech Res 25(5)2020. BJSTR. MS.ID.004277.

\section{ABSTRACT}

The presented material considers the possibility of combining sparingly soluble polysaccharides with plant polysaccharides to increase the absorption of drugs with low bioavailability, as biologically active substances they can serve as a targeted carrier for the delivery of diagnostic and therapeutic agents, as well as enzymes, nucleic acids, vitamins or hormones, to certain cells, in particular hepatocytes (parenchymal liver cells). A method for preparing a composition of arabinogalactan and chitosan succinate is described, examples of its practical use in medicine, veterinary medicine and animal husbandry are given.

Keywords: Arabinogalactan; Chitosan Succinate; Biologically Active Substances; Medicinal Substances

\section{Opinion}

Long-term studies of polysaccharides of higher plants have shown that they have biological activity, and therefore can be widely used [1-3]. Most representatives of galactane-containing polysaccharides of higher plants are immunomodulators that activate the reticuloendothelial system (RES), increase the phagocytic index. Biological activity largely depends on the features of the fine structure of macromolecules, i.e., on the structure of all side chains, their location along the main chain, the conformation of macromolecules, and the mechanism of aggregate formation. A certain role in the manifestation of biological activity belongs to the localization of the polysaccharide in the plant cell [1]. It was shown [3-6] that hypertension from western larch can serve as a targeted carrier for the delivery of diagnostic and therapeutic agents, as well as enzymes, nucleic acids, vitamins or hormones, to certain cells, in particular hepatocytes (parenchymal cells liver. In model experiments, hypertension from larch showed high membranotropy [7]. Due to this, it can be used to increase the absorption of other drugs characterized by low bioavailability $[2,5,7]$. In this case, a complex is formed between the delivered agent and arabinogalactan capable of interacting with the asialoglycoprotein receptor of the cell [8]. The reason for the interaction of arabinogalactan with these receptors may be the high branching of the structure of $\mathrm{AH}$ macromolecules and the presence of numerous terminal galactose and arabinose groups in them [1].

The conjugates of $\mathrm{AH}$ and its degradation products with various drugs can find various medical applications [9-15]. Arabinogalactan is a promising synthon capable of reacting with mono- and bifunctional reagents. This paves the way for new commercially available water-soluble preparations. Their properties will be determined both by the properties of the polysaccharide itself and the introduced functional groups. Chemical modification products of $\mathrm{AH}$ are of significant interest both for medicine and for various other industries. There is evidence that chemical modification of AG causes a noticeable decrease or disappearance of the bioactivity of polymers $[16,17]$. However, work on the modification of arabinogalactans show the promise of this direction. Based on the above statements, we decided to conduct studies on the combination of arabinogalactan polysaccharides with chitosan polysaccharides. The structural feature of AG macromolecules can contribute to the formation of strong intermolecular complexes of active substances, the molecules of which are most likely to be bound by intermolecular hydrogen bonds in the space formed by the side chains. 
Given the conformational mobility of AG macromolecules, the dimensions of this space can vary, contributing to the formation of supramolecular complexes with a wide range of substances [5]. Chitosan is a good gelling agent and emulsifier. Its ability to form complex compounds with proteins and lipids has been proven. The presence of high electron density hydroxyl groups with lone electron pairs along the molecule leads to the formation of hydrogen bonds with other biopolymer compounds; first of all, strong complexes with lipids and proteins are formed in which chitosan acts as a nucleus [6]. Due to the positively charged molecules and the properties of the sorbent, chitosan binds strongly to negatively charged molecules located on the membranes of microbes (teichoic acids, phosphate and carboxylic groups). Fixation of membranes paralyzes their work, especially the transport of ions, nutrients, which leads to increased sensitivity of pathogens to various antibacterial substances. Useful and important features of chitosan are that it combines with any living tissue, has mucoadhesive properties, decomposes and opens up tight joints between cells. By using these properties, drug delivery through the mucous membrane can be significantly improved.

Protonated chitosan in the body breaks down with the help of lysozyme, turning into glucosamine and the conjugated acid base - succinate. Chitosan succinate is a salt of deacetylated chitin and succinic acid. Chitosan succinate is hypoallergenic, biocompatible, biodegradable. Thanks to deacetylation, chitosan acquires two important properties that determine the features and scope of its use. Chitosan succinate is highly soluble in water; its deacylated and substituted groups at $\mathrm{pH}<7$ acquire a positive charge, i.e. chitosan gel is a polycation, which accordingly binds to negatively charged molecules. The production of the biopolymer matrix is based on the mechanochemical activation of two polysaccharides: plant (arabinogalactan) and chitin (chitosan succinate). The method of mechanochemical activation allows the grinding of solids to a molecular level. As a result, the crushed particles form aggregates, and with continued mechanical activation, mixing of solids occurs at the molecular level. In this case, depending on the nature of the substances, chemical reactions take place with the formation of a solid phase in which molecules enter into various kinds of interactions.

Further, during thermal exposure or hydration, a chemical reaction quickly occurs with the formation of target products. As a result, the crushed particles form aggregates, and with continued mechanical activation, mixing of solids occurs at the molecular level. In this case, depending on the nature of the substances, chemical reactions take place with the formation of a solid phase in which molecules enter into various kinds of interactions. Further, during thermal exposure or hydration, a chemical reaction quickly occurs with the formation of target products. The process of obtaining a polysaccharide matrix takes place in two stages. At the first stage, chitosan succinate was obtained by deacetylation of chitin having a deacetylation degree of greater than or equal to $75 \%$ and a molecular weight in the range from $1 \mathrm{kDa}$ to $30 \mathrm{kDa}$, and it was cross-linked using succinic anhydride mechanochemically. As a result of mechanical activation, quantitative acylation of all amino groups of chitosan occurs, which is confirmed by conductometric titration. It is significant that the very principle of carrying out the reaction in the solid-state state does not require the dissolution of dicarboxylic acid anhydrides in an organic solvent.

At the second stage, for the modification of arabinogalactan, the obtained chitosan succinate was mechanically coupled at the molecular level with arabinogalactan. Mechanochemical processing of the starting components was carried out in a rotary (roll) mill VM-1 with a drum having a fluoroplastic lining. The obtained fine powder was dissolved in water and the mixture was neutralized with an aqueous alkaline solution and dried by spray or vacuum drying [7]. The polymer matrix acquires a plastic structure of chitosan particles, characterized in that it has the form of a crosslinked cross-linked polymer with many spherical cavities having a size of not less than $1.0 \mathrm{~nm}$ and not more than $5000.0 \mathrm{~nm}$. The proposed composition of the biopolymer matrix includes chitosan succinate, succinic anhydride and arabinogalactan containing dihydroquercetin covalently unrelated to it. The complexing ability of the supramolecular composition: chitosan succinate, succinic anhydride, arabinogalactan, was studied by optical spectroscopy in order to assess the degree of increase in the solubility of hydrophobic drug compounds in water. Two compounds, carvedilol and zeaxanthin, which have a low intrinsic solubility in water, were taken for the study.

Zeaxanthin is one of the common antioxidants and pigments of the carotenoid group. Zeaxanthin is one of two carotenoids that are found in the retina (macular zone). As an antioxidant, it is able to neutralize free radicals that oxidize and damage the retina, which means it prevents the development of cataracts and serves as a preventive measure for age-related macular degeneration. One of the main limitations of the use of zeaxanthin in pharmacology and the food industry is its extremely low solubility in water. It was found that complexation with more than 1000 times increases the solubility of zeaxanthin in water. The concentration of the zeaxanthin - BPM complex 1:10 measured by us was 40 micromoles or $22 \mathrm{mg} / \mathrm{L}$. The measured increase in solubility (1000 times) is a lower estimate, since its intrinsic solubility is below the sensitivity limit of the device. Carvedilol is an antianginal, antioxidant, hypotensive, vasodilating drug. Slightly soluble in water. Bioavailability is about $25 \%$. It was found that complexation with a polymer matrix increases the solubility of carvedilol in water by 40 times. The concentration of the carvedilol-BPM complex 1:10 measured by us was 50 micromoles or $20 \mathrm{mg} / \mathrm{L}$. The absolute value of solubility was calculated from the measured extinction coefficient of carvediol at a wavelength of $332 \mathrm{~nm}$ equal to 4760 $1 \mathrm{~mol}^{-1} \mathrm{~cm}^{-1}$. A group of employees of the institutes of the SB RAS developed and tested anthelmintic drugs against operstachiasis, fascioliasis, etc. A 10-fold decrease in the active substance from 
the initial drug in combination with polysaccharides allows one to obtain a therapeutic effect of $95-100 \%$. A positive preventive effect was obtained on weaned pigs: good preservation and high weight gain when $10 \mathrm{~g}$ per head of polysaccharide composition was added to the feed.

\section{Conclusion}

Thus, studies have confirmed that the polysaccharide matrix has high biological activity both independently and in combination with other substances. Developed biologically active biopolymer matrix, including wt. \%: chitosan succinate 3.0-5.0; succinic anhydride 2.0-4.0; arabinogalactan 15.0-20.0; digiroquercetin 3-5 has a wide range of biologically active substances, has a fairly wide scope due to its high bioavailability, and the absence of side effects, which significantly expands the arsenal of using biologically active compositions in combination with a polysaccharide matrix.

\section{References}

1. Antonova GF, Tyukavkina NA (1976) Obtaining high-purity arabinogalactan from larch wood. Wood Chemistry 4: 60-62.

2. Arifkhodzhaev AO (2000) Galactans and galactan-containing polysaccharides of higher plants. Chemistry of Natural Compounds 3: 229-244.

3. Babkin VA, Ostroukhova LA, Malkov Yu A, Ivanova SZ, Onuchina NA, et al. (2001) Biologically active substances from larch wood. Chemistry in the interests of sustainable development 9(3): 363-367.

4. Grishin AA, Chitin, Chitosan (2014) Chemistry biological activity application News of universities. Applied Chemistry and Biotechnology 6(1): 29-34.

5. Ovodov Yu S (1998) Polysaccharides of flowering plants: structure and physiological activity. Bioorganic chemistry 24(7): 483-501.

ISSN: 2574-1241

DOI: $10.26717 /$ BJSTR.2020.25.004277

Shelepov VG. Biomed J Sci \& Tech Res

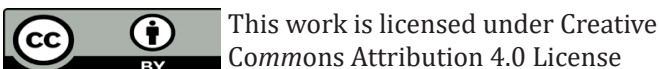

Submission Link: https://biomedres.us/submit-manuscript.php
6. Fazilova SA (2010) Structural studies of polysaccharides and nanocomposites based on them. SA Fazilova, SM Yugay, S Rashidova Chemistry of plant raw materials 1: 13-19.

7. Shelepov VG, Uglov VA, Dushkin AV, Suntsova LP, Boroday EV, etal. (2019) Biopolymein matrix based on chitosan arabinogalactan succinate and method for its preparation. Pat. RUS No. 2698455, publ. 08/27/2019.

8. Assam T Eliyahu, Shapira L, Linial M, Barenholz Y, Domb et al. (2002) Polysaccharide-oligoamine based conjugates for gene delivery. Journal Med Chem 45(9): 1817-1824.

9. Chintalwar G, Jain A, Sipahimalani A, Banerji A, Sumariwalla P, et al. (1999) An immunologically active arabinogalactan from Tinospora cordifolia. Phytochemistry 52(6): 1089-1093.

10. Chun Ho Ming (2002) Conditioning shampoo containing arabinogalactan. Pat 6406686 US CA 137: 10711.

11. Silva BP, Parente JP (2002) Chemical properties and biological activity of a polysaccharide from Melocactus depressus. Planta Medica 68(1): 74-76.

12. Dahl K (1967) Recovery of high-purity arabinogalactan from larch. Pat US 3509126 CA 100269.

13. Clarcke AE, Anderson RL, Stone BA (1979) Form and function of arabinogalactans and arabinogalactan-proteins. Phytochemistry 18: 521-540.

14. Da Silva BP, Parente JP (2002) Chemical properties and biological activity of a polysaccharide from Melocactus depressus. Planta Medica 68(1): 74-76.

15. Grieshop CM, Flickinger EA, Fahey GC (2002) Oral administration of arabinogalactan affects immune status and fecal microbial populations in dogs. Journal of Nutrition 132(3): 478-482.

16. Groman EV, Enriquez PM, Jung Chu, Josephson L (1994) Arabinogalactan for hepatic drug delivery. Bioconjugate Chem 5: 547-556.

17. Sajjad A (2017) Chitosan as a Flocculant: An Approach to Improve its Solubility for Efficient Harvesting of Microalgae. Korean Chemical Engineering Research 55(4): 530-534.

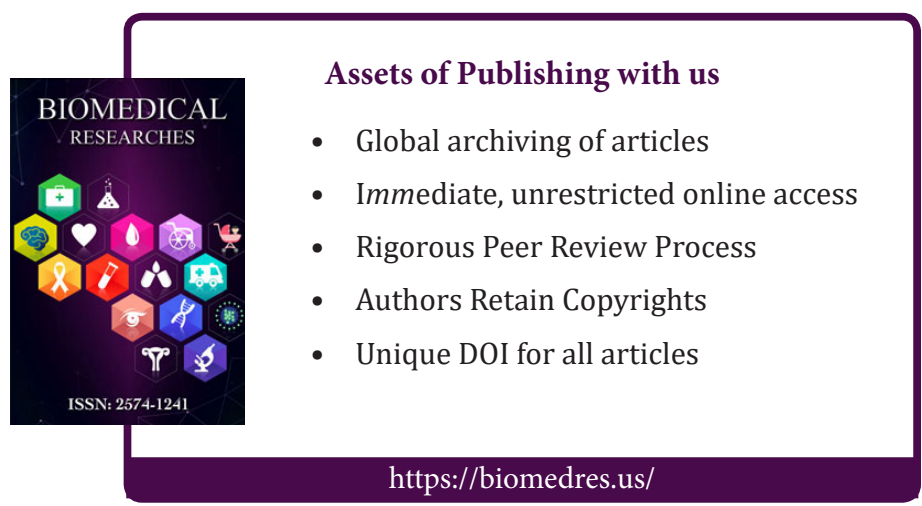

